\title{
Miniature optical delay lines and buffers
}

\author{
Artemiy V. Dmitriev ${ }^{(1)}$, Nikita A. Toropov ${ }^{(2)}$ and M. Sumetsky ${ }^{(1)^{*}}$, \\ ${ }^{(1)}$ Aston Institute of Photonic Technologies, Aston University, Birmingham B4 7ET, UK \\ ${ }^{(2)}$ ITMO University, St.Petersburg, 197101, Russia \\ *e-mail: m.sumetsky@aston.ac.uk
}

\begin{abstract}
Creation of miniature optical delay lines and buffers is one of the greatest challenges of the modern photonics which can revolutionize optical communications and computing. Several remarkable designs of slow light optical delay lines employing coupled ring resonators and photonic crystal waveguides has been suggested and experimentally demonstrated. However, the insertion loss of these devices is too large for their practical applications. Alternatively, the recently developed photonic fabrication platform, Surface Nanoscale Axial Photonics (SNAP) allows us to fabricate record small delay lines with unprecedentedly small dispersion and low loss. In this report, we review the recent progress in fabrication and design of miniature slow light devices and buffers, in particular, those based on the SNAP technology.
\end{abstract}

Keywords: microresonators, nanophotonics, optical delay lines, optical buffers

\section{MICROSCOPIC OPTICAL DELAY LINES AND BUFFERS}

Microscopic optical delay lines and buffers are expected to be the key elements of the future optical communication and computing technologies. Creation of these elements is considered as one of the greatest challenges of the modern photonics due to the required minimum possible dimensions, attenuation of light, and

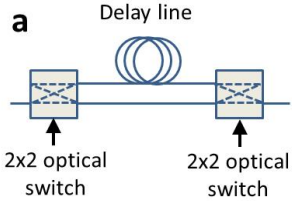

b

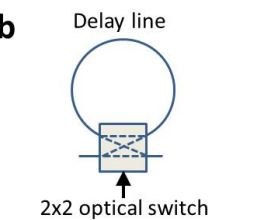

Figure 1. Illustration of a feedforward optical buffer (a) and a feedback optical buffer (b). switching power. It is usually assumed that the principle structure of microscopic optical buffers will remain similar to that of the macroscopic optical buffers illustrated in Fig. 1 [1,2]. This figure shows buffers with two major functionalities: a feedforward buffer, which includes an open delay line and two 2x2 switches (Fig. 1(a)), and a feedback buffer, which includes a recirculation loop delay line and a $2 \times 2$ switch (Fig. 1(b)). Similar to macroscopic buffers, microscopic buffers should trap optical pulses, hold them for the required period of time, and release without distortion. The key element of a microscopic buffer is a delay line, which is required to have the smallest possible dimensions and losses. Miniature optical delay lines proposed and experimentally demonstrated to date were based on the concept of slow light, which employ propagation of light in engineered structures, e.g., coupled ring resonators and photonic crystals, where the propagation of light is slow due to multiple reflections and circulations [3-6]. Yet, practical miniature slow light delay lines have not been fabricated due to insufficient precision of modern photonics technologies and attenuation of light $[2,7]$.

\section{SNAP DELAY LINES AND BUFFERS}

On the other hand, the recently developed Surface Nanoscale Axial Photonics (SNAP) platform allows to fabricate miniature slow light structures with unprecedentedly high precision and low loss [8-11]. The SNAP structures are introduced at the surface of an exceptionally low loss and smooth optical fiber by nanoscale deformation. In [10], the ultralow loss miniature parabolic bottle resonator delay line, which fabricated with a subangstrom precision in effective radius variation (ERV) of an optical fiber, was experimentally demonstrated. In [11], it was shown that fully reconfigurable SNAP photonic circuits can be introduced at the surface of an optical fiber with subangstrom precision by local heating. In [12], a miniature bottle resonator optical buffer based on a tunable version of the delay line demonstrated in [10] was proposed. The performance of this device, which is similar to that of the feedback optical buffer in Fig. 1(b), is illustrated in Fig. 2. Light is coupled in and out of the bottle resonator through a microfibre waist of a transverse biconical optical taper (Fig. 2(a)). The bottle

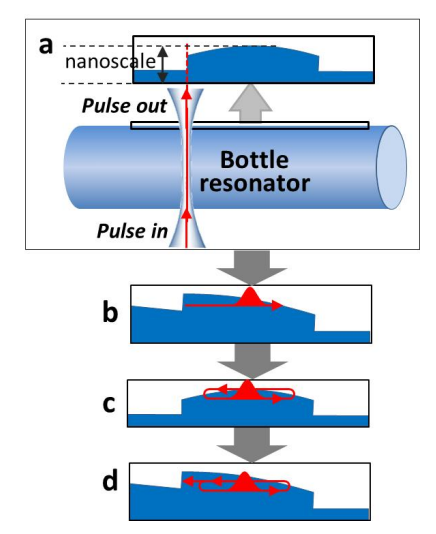

Figure 2. Illustration of a bottle resonator optical buffer (a) and its opening to aquire a pulse (b), closing to trap a pulse and closing to release a pulse (c). 
resonator can be opened by nanoscale ERV to let the optical pulse in (Fig. 2(b)). After the pulse is completely inside the resonator, the bottle resonator is closed (Fig. 2(c)) and the pulse dwells inside the resonator for the required period of time corresponding to the integer number of oscillation cycles. Finally, the resonator is opened (Fig. 2(d)) and the pulse is released. Due the semi-parabolic ERV profile of the bottle resonator, the pulse does not experience distortion over time [12] and its bandwidth and dwell time are not restricted by the delay timebandwidth limitation.

The described opening and closing of the buffer can be realized using a fiber with a highly nonlinear or piezoelectric core $[13,14]$. The application of a pico/nanosecond laser pulses [13] or electric pulses, can tune the ERV of the fiber and, thus, open and close the bottle resonator. As an example, Fig. 3 shows an optical buffer with a piezoelectric core. The tunability of this device can be realized with a microscopic piezoelectric transducer, which has been designed and theoretically investigated in the static regime in [15].

Since the absolutely precise parabolic potential cannot be

Figure 3. Illustration of a bottle resonator optical buffer driven by a speccially designed piesoelectric core. realized experimentally, the required fabrication precision of the SNAP buffer was numerically estimated [12]. To this end, the effects of stationary perturbations of parabolic potential, which disturb the periodicity of pulse oscillations, and the time-dependent perturbations, which affect the process of entering and exiting of the resonator, were investigated. It was found that perturbations, which are localized near the turning points of the pulse, have a much stronger effect than those localized in the middle of the bottle resonator and also those disturbing the entering and exiting processes. Overall, it was concluded that the fabrication precision of 0.1 angstrom in ERV of the semi-parabolic bottle resonator is required for the satisfactory performance of the proposed optical buffer.

\section{ULTRAPRECISE SNAP FABRICATION TECHNOLOGY}

Improvement of the fabrication precision is one of the most critical challenges of modern microphotonic technology, which will ensure its broad practical applications in optical communications, quantum computing, and ultraprecise optical measurements. Ultralow loss miniature optical delay lines and buffers can become key elements for many of these applications. The development of SNAP technology allowed to demonstrate the record subangstrom fabrication precision of microscopic photonic elements and circuits $[9,10]$.

Recently, we have fabricated two coupled SNAP bottle resonators with a better than 0.17 angstrom precision in the ERV (Fig. 4) [16]. Using postprocessing, we reduced the deviation of fundamental resonances 1 and 2 shown in this figure below the measurement resolution $1.3 \mathrm{pm}$, which corresponds to 0.17 angstrom in ERV. This an order of magnitude improvement of the precision achieved by post-processing in planar photonics technologies [18-21]. Since the precision achieved in [16] was limited by the resolution of the optical spectrum analyzer used, we suggest that the ultrahigh precision of $0.1 \AA$ required for the fabrication of a SNAP buffer described in Section 2 is feasible and can be achieved by modification of the SNAP fabrication setup.

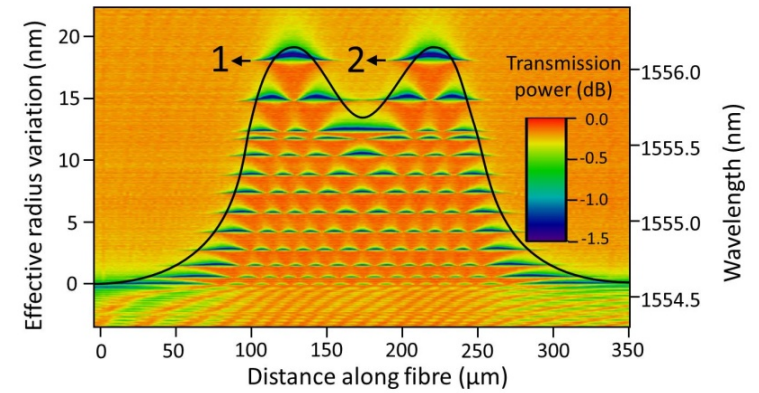

Figure 4. Experimentally measured surface plot of the resonance spectra of a double bottle resonator fabricated in $[167$.

\section{SUMMARY}

Based on our recent experimental and theoretical studies, we suggest that the future development of the SNAP platform, which includes the enhancement of the fabrication precision and enrichment with internal functionalities (e.g., using SNAP fibers with highly nonlinear and piezoelectric cores), will allow us to solve the long standing problem of creating a microscopic optical buffer, the key element of the future microphotonic devices with broad practical applications.

\section{ACKNOWLEDGEMENTS}

M. Sumetsky acknowledges the Royal Society Wolfson Research Merit Award. 


\section{REFERENCES}

[1] R. S. Tucker, P. C. Ku, and C. J. Chang-Hasnain, "Slow-light optical buffers: capabilities and fundamental limitations," J. Lightwave Technol. 23, 4046 (2005).

[2] E. F. Burmeister, D. J. Blumenthal, and J. E. Bowers, "A comparison of optical buffering technologies," Opt. Switching and Netw. 5, 10 (2008).

[3] F. N. Xia, L. Sekaric, and Y. Vlasov, "Ultracompact optical buffers on a silicon chip," Nat. Photon. 1, 65 (2007).

[4] T. Baba, "Slow light in photonic crystals," Nat. Photon. 2, 465 (2008).

[5] M. Notomi, "Manipulating light with strongly modulated photonic crystals," Rep. Prog. Phys. 73, 096501 (2010).

[6] M. F. Yanik and S. Fan, "Stopping light all optically," Phys. Rev. Lett. 92, 083901 (2004).

[7] W. Bogaerts, M. Fiers, and P. Dumon, "Design Challenges in Silicon Photonics," IEEE J. Sel. Topics Quantum Electron. 20, 1 (2014).

[8] M. Sumetsky, D. J. DiGiovanni, Y. Dulashko, J. M. Fini, X. Liu, E. M. Monberg, and T. F. Taunay, "Surface nanoscale axial photonics: robust fabrication of high-quality-factor microresonators," Opt. Lett. 36, 4824 (2011).

[9] M. Sumetsky and Y. Dulashko, "SNAP: Fabrication of long coupled microresonator chains with subangstrom precision," Opt. Express 20, 27896 (2012).

[10] M. Sumetsky, "Delay of Light in an Optical Bottle Resonator with Nanoscale Radius Variation: Dispersionless, Broadband, and Low Loss", Phys. Rev. Lett. 111, 163901 (2013).

[11] A. Dmitriev, N. Toropov, and M. Sumetsky, "Transient reconfigurable subangstrom-precise photonic circuits at the optical fiber surface," Postdeadline paper, 28th IEEE Photonics Conference (Reston, 2015); doi: 10.1109/IPCon.2015.7323759.

[12] M. Sumetsky, "Microscopic optical buffering in a harmonic potential," Scientific Reports 5, 18569 (2015).

[13] F. H. Suhailin, N. Healy, Y. Franz, M. Sumetsky, J. Ballato, A. N. Dibbs, U. J. Gibson, and A. C. Peacock, "Kerr nonlinear switching in a hybrid silica silicon microspherical resonator," Opt. Express 23, 17263 (2015).

[14] D. Homan, G. Kaur, G. Pickrell, B. Scott, and C. Hill, "Electronic and magnetic fibers," Mat. Lett. 133, 135 (2014).

[15] A. V. Dmitriev and M. Sumetsky, "Tunable photonic devices at the surface of an optical fiber with piezoelectric core," Opt. Lett. 41, 2165 (2016).

[16] N. A. Toropov and M. Sumetsky, "Permanent matching of coupled optical bottle resonators with better than $0.16 \mathrm{GHz}$ precision," Opt. Lett. 41, 2278 (2016).

[17] H. Lee, T. Chen, J. Li, K. Y. Yang, S. Jeon, O. Painter and K. J. Vahala, "Chemically etched ultrahigh-Q wedge-resonator on a silicon chip," Nat. Photon. 6, 369 (2012).

[18] T. Barwicz, M. A. Popovic, M. R. Watts, P. T. Rakich, E. P. Ippen, and H. I. Smith, "Fabrication of adddrop filters based on frequency-matched microring resonators," J. Lightw. Technol. 24, 2207 (2006).

[19] V. Krishnamoorthy, X. Zheng, G. Li, J. Yao, T. Pinguet, A. Mekis, H. Thacker, I. Shubin, Y. Luo, K. Raj, and J. E. Cunningham, "Exploiting CMOS Manufacturing to Reduce Tuning Requirements for Resonant Optical Devices," IEEE Photonics J. 3, 567 (2011).

[20] S. Prorok, A. Yu. Petrov, M. Eich, J. Luo, and A. K.-Y. Jen, "Trimming of high-Q-factor silicon ring resonators by electron beam bleaching," Opt. Lett. 37, 3114 (2012).

[21] P. Alipour, A. H. Atabaki, M. Askari, A. Adibi, and A. A. Eftekhar, "Robust postfabrication trimming of ultracompact resonators on silicon on insulator with relaxed requirements on resolution and alignment," Opt. Lett. 40, 4476 (2015). 